\title{
„Ende Juli. Eine Fliege stirbt: Weltkrieg“. Zu Robert Musils Wahrnehmung des Krieges
}

"Fin juillet. Une mouche meurt: guerre mondiale». Sur la perception de la guerre par Robert Musil

"End of July. A fly dies: World War". How Robert Musil perceives the War

\section{Claus Erhart}

\section{(2) OpenEdition}

\section{Journals}

Édition électronique

URL : http://journals.openedition.org/ceg/2027

DOI : $10.4000 /$ ceg. 2027

ISSN : 2605-8359

Éditeur

Presses Universitaires de Provence

Édition imprimée

Date de publication : 15 juin 2014

Pagination : 135-149

ISSN : 0751-4239

Référence électronique

Claus Erhart, " Ende Juli. Eine Fliege stirbt: Weltkrieg“. Zu Robert Musils Wahrnehmung des Krieges », Cahiers d'Études Germaniques [Online], 66 | 2014, Online erschienen am: 17 Dezember 2017, abgerufen am 10 Dezember 2020. URL : http://journals.openedition.org/ceg/2027 ; DOI : https://doi.org/ 10.4000/ceg.2027 


\title{
„Ende Juli. Eine Fliege stirbt: Weltkrieg“. Zu Robert Musils Wahrnehmung des Krieges
}

\author{
Claus ERHART \\ Université Nice Sophia Antipolis
}

\section{Kriegseuphorie}

In einem Artikel über die internationale Friedensbewegung schildert der früh verstorbene Wiener Kulturhistoriker Albert Fuchs (1905-1946) die geistige Lage seiner Heimat in den „Sommermonaten 1914“. Damals

\begin{abstract}
begann eine Überschwemmung des österreichischen Volkes mit chauvinistischer Propaganda. Alle politischen Parteien, einschließlich der Arbeiterpartei, waren an dieser Propaganda beteiligt. Sie hatten durch Jahre und Jahrzehnte das Gift der deutsch-imperialistischen Ideologie in sich aufgenommen, nun brach es wie eine ekelerregende Krankheit aus ihnen heraus. [...] Wesentliche Teile der Intelligenz wurden, durch die Kundgebungen der Regierung, durch den Taumel der Massen beeindruckt, mitgerissen. Ärzte und Musiker, Nationalökonomen und Philosophen wetteiferten in Zeitungsartikeln und Deklarationen, ihre Unfähigkeit zu selbständigem Denken zu erweisen. Besonders schlimme Verheerungen gab es im Felde der Literatur.
\end{abstract}

Wenn in der Liste prominenter Autoren, die laut Fuchs zu diesen „Verheerungen“ beigetragen haben, der Name Robert Musils fehlt, dann wohl auch darum, weil dessen Euphorie rascher abgeklungen war als die vieler seiner Schriftsteller-Kollegen. Es weist dieses Fehlen aber auch auf eine Tendenz zur Exkulpation des Autors voraus, die in der Sekundärliteratur späterer Jahre unschwer nachgewiesen werden kann. Die verschwommene Vorstellung, Musils anfängliche Kriegsbegeisterung habe sich vor allem aus der Hoffnung genährt, der Krieg werde den Unzulänglichkeiten im geistigen und politischen Leben seiner Zeit ein Ende bereiten², greift ebenso zu kurz

\footnotetext{
${ }^{1}$ Albert FUCHS, „Pazifismus“, in Albert FuCHS, Geistige Strömungen in Österreich: 1867 1918, Nachdruck der Ausgabe 1949. Mit einem Vorwort von Friedrich Heer. Wien, Löcker, 1984, S. 270

Vgl. Wilfried Berghahn, Robert Musil in Selbstzeugnissen und Bilddokumenten, Reinbek bei Hamburg, Rowohlts Monographien, 1972, S. 71.
} 
wie die These, wonach seine Faszination angesichts des beginnenden Konflikts sich zwanglos und zwangsläufig aus seinem Dichtungsbegriff herleiten lasse ${ }^{3}$. In diese letztere Perspektive gerückt, erscheint Musil als eine Art Hohepriester der Literatur, der in der Mobilisierung gleichsam nur die Fortsetzung seiner Poetik mit anderen Mitteln gesehen habe. ${ }^{4}$ Stichwortgeber für solch milde Deutungen war Musil selbst, etwa mit der Formel, Kunst sei, wo sie „Wert" habe, „erobernd, nicht pazifizierend“" Kriegsausbruch formulierten Vorstellung, große Kunst werde „von dem gleichen kriegerischen und erobernden Geist belebt, den wir heute in seiner Urart verwundert und beglückt in uns und um uns fühlen" (PS, 1021).

Will man sich ein genaueres Bild von Musils Beiträgen zur Beurteilung der Situation im Sommer 1914 machen, so verdient vor allem der Essay Europäertum, Krieg, Deutschtum Beachtung, der zusammen mit Texten u.a. von Richard Dehmel, Gerhart Hauptmann, Alfred Kerr oder Emil Ludwig im Septemberheft der Neuen Rundschau erschienen war. „Es muß damals“, räumt Musil-Biograph Karl Corino ein, „ungeheuer schwer gewesen sein, sich der kollektiven Psychose zu entziehen."6 Dennoch fällt er über Musils Aufsatz ein unmissverständlich hartes Urteil: Es war dieser

Tribut an den Zeitgeist, an die Zeitgeistkrankheit [...] ein Gegenstück zu der Kriegsbegeisterung, wie man sie aus den Manifesten der italienischen Futuristen kannte, und in den Augen der Nachgeborenen zugleich eines der deprimierendsten Beispiele aus der Musilschen Publizistik. ${ }^{7}$

Tatsächlich lieferte der Autor, bevor es ins Feld zu ziehen galt, in diesem Essay auf knappem Raum sein ,geistiges Testament“ (PS, 1020), das sich von vielen ähnlichen Vermächtnissen der Zeit nur durch geschliffenere Formulierungen unterscheidet und durch die bereits erwähnte Engführung des in der hohen Literatur und in der Mobilisierung sich artikulierenden Geistes. Davon einmal abgesehen, bemüht der Text die gleichen patriotischen, nationalistischen und soldatischen Klischees, denen ein Deutscher, Österreicher oder auch Russe, Engländer oder Franzose im ersten Kriegsjahr notgedrungen ausgesetzt war: Die Schuld am Konflikt tragen die anderen, die in einer „Verschwörung unsere Ausrottung beschlossen haben“, der „Einzelne“ geht vollkommen auf in „seiner elementaren Leistung, den Stamm zu schützen“, der Krieg ist „schön und brüderlich“, der „Tod hat keine Schrecken mehr" und die, ,welche sterben müssen oder ihren Besitz opfern,

\footnotetext{
3 Vgl. Hertwig Gradischnig, Das Bild des Dichters bei Robert Musil, Musil-Studien, Bd. 6, München/ Salzburg, Wilhelm Fink, 1976, S. 24 f.

${ }^{4} \mathrm{Vgl}$. Zu dieser Tendenz in der Sekundärliteratur auch Alexander HONOLD, Die Stadt und der Krieg. Raum- und Zeitkonstruktionen in Robert Musils Roman „Der Mann ohne Eigenschaften “, Musil-Studien, Bd. 25, München, Wilhelm Fink, 1995, S. 207, Anm. 31.

${ }_{5}^{5}$ Robert MUSIL, Prosa und Stücke, Kleine Prosa, Aphorismen, Autobiographisches, Essays und Reden, hrsg. von Adolf Frisé, Reinbek bei Hamburg, Rowohlt, 1978. Zitatbelege künftig nach dieser Ausgabe im Text (Sigle: PS + Seitenzahl), hier: PS, 981.

${ }^{6}$ Karl CORINO, Robert Musil. Eine Biographie, Reinbek bei Hamburg, Rowohlt, S. 492.

7 Ibid., S. 493.
} 
haben das Leben und sind reich" (vgl. PS, 1020-1022). Der Autor, der noch im November 1913 in den Weißen Blättern geschrieben hatte:

Ich hielt, dass alle Menschen im Grunde gleich und Brüder seien, immer für eine sentimentale Übertreibung und tue es noch heute, denn mein Gefühl wurde von den anderen stets mehr abgestoßen als angezogen. (PS, 1011)

- derselbe Autor sieht sich nun vom Gefühl „eine[r] betäubende[n] Zugehörigkeit“ in sein „Volk hineingerissen“ (PS, 1021). Dieser offensichtliche Wandel in der Anschauung ist ein Hinweis auf die Richtigkeit der These Walter Benjamins, wonach es ,nur der Krieg [...] möglich [macht], Massenbewegungen größten Maßstabs unter Wahrung der überkommenen Eigentumsverhältnisse ein Ziel zu geben." ${ }^{8}$ Dass der proklamierten Verbrüderungseuphorie im Empfinden Musils dennoch soziale und kulturelle Grenzen gesetzt waren, wird aus einem späteren Tagebucheintrag ersichtlich, in dem er sich daran erinnert, als Soldat „plötzlich von lauter Menschen umgeben“ gewesen zu sein, „die nie ein Buch lasen“. Das Resümee dieser Erfahrung - er spricht immerhin von „einem der stärksten alten Kriegseindrücke“ - fällt etwas prätentiös aus, wenn es heißt: „Welche unerwartete u. breite Berührung mit dem Durchschnittsleben!‘ 99

Wenn in Musils Bewusstsein nach ein „ein paar Wochen ethischen Aufschwungs" (T, 354) auch wieder Ernüchterung eingekehrt war, wäre es doch verfehlt, in dem zitierten Essay einfach nur eine verspätete Jugendsünde des damals 34-jährigen Autors zu sehen. Tatsächlich trug er die Faszination für die Ereignisse um den Ausbruch des 1. Weltkrieges, die sein Menschenbild wesentlich geprägt haben, für den Rest seines Schriftstellerlebens mit sich. Er sah im Begeisterungsfieber um die Mobilisierung ein religiöses (vgl. etwa T, 339, 540, 544) oder „dem religiösen verwandtes Erlebnis“ (PS, 1060), dessen Ursachen und Voraussetzungen er in den Jahren nach 1918 zu ergründen suchte ${ }^{10}$. In einem Essay aus dem Jahre 1921 stellt er fest:

Man führt ja [...] den Weltkrieg [...] bald auf diese, bald auf jene Ursachengruppe zurück. Aber das ist Täuschung. Ebensolcher Schwindel, wie wenn man ein einzelnes physisches Ereignis auf eine Ursachenkette zurückführt. In Wirklichkeit zerfließen die Ursachen schon bei den ersten Gliedern der Kette in eine unabsehbare Breite. (PS, 1056 f.)

\footnotetext{
${ }^{8}$ Walter BENJAMIN, „Das Kunstwerk im Zeitalter seiner technischen Reproduzierbarkeit“, in Walter BENJAMIN, Illuminationen. Ausgewählte Schriften 1, Frankfurt am Main, Suhrkamp, 1977, S. 168

${ }^{9}$ Robert MusiL, Tagebücher, hrsg. von Adolf Frisé, Reinbek bei Hamburg, Rowohlt, 1976. Zitatbelege künftig nach dieser Ausgabe im Text (Sigle: T + Seitenzahl), hier: T, 945.

${ }_{10}$ Ein Überblick über Musils weitläufige Überlegungen zum Thema Weltkrieg findet sich etwa in Paul ZÖCHBAUER, Der Krieg in den Essays und Tagebüchern Robert Musils, Stuttgarter Arbeiten zur Germanistik, Nr. 316, Unterreihe Salzburger Beiträge, Nr. 25, Stuttgart, Heinz, 1996, vor allem S. 44-62.
} 
Die „unabsehbare Breite“ ist ein früher Hinweis auf den Mann ohne Eigenschaften, in dem Musil den Versuch unternehmen wird, den Ausbruch des Krieges als Ergebnis all der im Roman gezeigten heterogenen Strömungen, Bewegungen und Bewusstseinslagen darzustellen. Meine eigenen Absichten die folgenden Bemerkungen betreffend sind naturgemäß wesentlich bescheidener. Dass das hoch flammende Pathos von Musils Kriegsapologetik erlöschen würde, war eine Frage der Zeit und hängt wohl mit der Anschaulichkeit zusammen, die jeder Realität innewohnt, auch oder gerade der Realität bewaffneter Konflikte. Dennoch scheint der Prozess der Desillusionierung einer Logik zu folgen, die an die Besonderheiten des 1. Weltkriegs geknüpft sein dürfte und die ich anhand der wenigen Texte, die der Autor zwischen 1914 und 1918 verfasst hat, im Folgenden erläutern möchte.

\section{Kriegserlebnis}

Noch in Berlin, im August 1914, hatte Musil erkannt, dass angesichts der sich überstürzenden Ereignisse „Psychotiker [...] in ihrem Element“ waren, und als die ersten Verlustlisten eintrafen, vermerkte er im Tagebuch seine Erschütterung darüber: ,[...] tot ... tot ... tot ... so untereinandergedruckt, niederschmetternder Eindruck.“ (T, 299)

Musil rückt Ende August zum Landsturm nach Linz ein und wird Anfang September mit seiner Kompanie zum „ausübenden Grenzschutz“ nach Südtirol abkommandiert. ${ }^{11}$ Als Italien am 23. Mai 1915 Österreich-Ungarn den Krieg erklärt, verwandelt sich die zu schützende Grenze in eine Kampfzone und aus dem monatelang geübten harmlosen „Pfadfinderspiel““12 wird mit einem Schlag blutiger Ernst. Bis zum Kriegsende wird Musil etwa eineinhalb Jahre an der Front verbringen und unter anderem an vier Isonzoschlachten teilnehmen. Am Isonzo, dem heutigen Grenzgebiet zwischen Italien und Slowenien, war ein großer Teil der italienischen Armee stationiert. Die Front erstreckte sich über $64 \mathrm{~km}$, im Laufe der insgesamt 12 Schlachten, die sich Österreicher und Italiener lieferten, gab es auf beiden Seiten zusammen etwa eine Million Tote und Verwundete. ${ }^{1}$

Wer wie Musil das amtliche Zeugnis ausgestellt bekam, am Isonzo „Vorzügliches geleistet“ und ,im feindlichen Artilleriefeuer [...] schwierige Rekognoszierungen [...] mit Geschick bewirkt" zu haben, ${ }^{14}$ der wusste also, wenn das Thema auf den Krieg kam, wovon er sprach, oder besser: wovon er schwieg. Denn es ist in der Tat erstaunlich, in welch hohem Maße Walter Benjamins Beobachtung, dass die Menschen stumm aus dem Krieg zurückkehrten, auch auf einen Mann zutrifft, der seit 1910 die

\footnotetext{
11 Vgl. CORINO, Musil, S. 497.

12 Ibid., S. 520.

13 Vgl. ibid., S. 543

${ }^{14}$ Vgl. ibid., S. 545 f.
} 
Berufsbezeichnung Schriftsteller führte und der nicht im Verdacht stehen kann, sich seinen prominenten Platz in der Literaturgeschichte durch Einsilbigkeit erworben zu haben. Im Aufsatz Erfahrung und Armut aus dem Jahre 1933 schreibt Walter Benjamin:

[...] die Erfahrung ist im Kurs gefallen und das in einer Generation, die 19141918 eine der ungeheuersten Erfahrungen der Weltgeschichte gemacht hat. Vielleicht ist das nicht so merkwürdig wie das scheint. Konnte man damals nicht die Feststellung machen: die Leute kamen verstummt aus dem Felde? Nicht reicher, ärmer an mitteilbarer Erfahrung. ${ }^{15}$

Drei Jahre später führt er den Gedanken im Essay Der Erzähler ergänzend weiter. Dieses Verstummen sei in der Tat

nicht merkwürdig. Denn nie sind Erfahrungen gründlicher Lügen gestraft worden als die strategischen durch den Stellungskrieg, die wirtschaftlichen durch die Inflation, die körperlichen durch die Materialschlacht, die sittlichen durch die Machthaber. Eine Generation, die noch mit der Pferdebahn zur Schule gefahren war, stand unter freiem Himmel in einer Landschaft, in der nichts unverändert geblieben war als die Wolken und unter ihnen, in einem Kraftfeld zerstörender Ströme und Explosionen, der winzige, gebrechliche Menschenkörper. ${ }^{16}$

Einen ganz ähnlichen Gedanken findet man in Musils 1922 geschriebenem Essay Das hilflose Europa, wenn er auf die Kriegsjahre zurückblickend meint:

[...] wir waren früher betriebsame Bürger, sind dann Mörder, Totschläger, Diebe, Brandstifter und ähnliches geworden: und haben doch eigentlich nichts erlebt. [...] wir haben viel gesehen und nichts wahrgenommen.

Darauf gibt es, glaube ich, nur eine Antwort: Wir besaßen nicht die Begriffe, um das Erlebte in uns hineinzuziehen. [...] Zurückgeblieben ist nur eine sehr erstaunte Unruhe, ein Zustand, als hätten sich vom Erlebnis her Nervenbahnen zu bilden begonnen und wären vorzeitig abgerissen worden. (PS, 1075 f.)

Erlebnisse, die sich in dem angegebenen Sinn ohne die entsprechende Unterstützung durch die Begriffe nicht zur Wahrnehmung entwickeln kantisch: Anschauungen, die ohne Begriffe blind bleiben - ziehen sich wie ein roter Faden durch Musils spärliche Tagebucheintragungen aus den Kriegsjahren. Im Juli 1915 notiert er etwa:

Beschießung des Werks Mte Verena durch 30.5 Mörser gesehn. Wo das Geschoß einschlägt[,] steigt senkrecht eine Fontäne von Rauch und Staub auf, die oben wie eine Pinie breit wird. Man hat ein neutrales Gefühl wie beim Scheibenschießen.

[...] Eine italienische Batterie schwerer Geschütze sucht mit Schrapnells unsren Mörser. Staubwolke in der Luft, kein anderer Eindruck. [Nachtrag:] Helle Rauchwolke. (T, 309)

\footnotetext{
${ }^{15}$ Walter BENJAMIN, „Erfahrung und Armut“, in Illuminationen, S. 291.

16 Walter BENJAMIN, „Der Erzähler. Betrachtungen zum Werk Nikolai Lesskows“, in Illuminationen, S. 386
} 
Es ist manchmal schwer zu entscheiden, ob der dem Ereignis beiwohnende und dieses Ereignis festhaltende Tagebuchschreiber die Begriffsbildung nicht leisten kann oder nicht leisten will. Der $30,5 \mathrm{~cm}-$ Mörser, der in der k.u.k. Armee ab 1914 zum Einsatz kam, wog an die 19 Tonnen. Er verschoss bei einer Reichweite von 9,6 Kilometern $385 \mathrm{~kg}$ schwere Sprenggranaten, die zwar imstande waren, zwei Meter starken Beton zu durchschlagen ${ }^{17}$, offenbar aber nicht den Panzer, hinter dem in dieser Tagbuchnotiz ein neutrales „Man [...] ein neutrales Gefühl“ registriert und Assoziationen signalisiert (,Scheibenschießen“), die mehr an einen Sonntagsausflug als an Krieg denken lassen. Dass sich bei einem Angriff durch schwere feindliche Geschütze außer zwei bescheidenen optischen Wahrnehmungen („Staubwolken in der Luft“, „Helle Rauchwolke“) kein anderer Eindruck einstellen will, kann vielleicht als Beleg für die „,vorzeitig abgerissen[en] [...] Nervenbahnen“ im Sensorium des Beobachters gelten. Jedenfalls bleibt hier mit der Extrembelastung für das Gehör zumindest ein wesentliches Element ausgespart, das in Schilderungen vergleichbarer Gefechtssituationen in Kriegsreportagen, aber auch in der medizinischen Literatur der Zeit regelmäßig wiederkehrt ${ }^{18}$.

Nach einigen Betrachtungen erotischer Natur über die Bäuerinnen der Gegend, die später zum Teil wörtlich in die Erzählung Grigia einfließen werden, folgt eine Eintragung, die deutlich macht, dass der „niederschmetternde Eindruck“, der sich noch in Berlin angesichts der gedruckten Verlustlisten eingestellt hatte (vgl. T, 299), nun einer distanzierteren Haltung gewichen ist: „Die Gefechte, Toten usw., die sich vor den Stellungen abspielten, haben mir bisher keinen Eindruck gemacht.“ (T, 312)

Auch hier wird ausdrücklich betont, dass die Eindrücke eigentlich keinen Eindruck machen, sondern an der Netzhaut des unbeteiligten oder sich unbeteiligt gebenden Augenzeugen abgleiten.

Sind Tod und Sterben ein Thema, an dem sich schon unter normalen Lebensbedingungen auf Dauer nicht vorbeidenken lässt, so entwickeln sie in den Kampfhandlungen des 1. Weltkriegs eine empirische Aufdringlichkeit, der sich auch Musils widerspenstiges Tagebuch-Ich nicht ganz entziehen kann. Die Gegenwehr, die es dabei aber dennoch leistet, wird als Verschiebung sichtbar, und zwar zunächst als Konzentration der Aufmerksamkeit auf das Leiden und Sterben der nicht menschlichen Kreatur. „Ende Juli. Eine Fliege stirbt: Weltkrieg“ (T, 309), lauten die ersten Worte einer Notiz, in der die Agonie einer von einem Fliegenpapier auf den Tisch gefallenen Fliege beschrieben wird: „Ihre sechs Beinchen legen sich

${ }^{17}$ Vgl. http://www.fl18.de/artikel/319/ (aufgerufen am 31.10.2013).

8 Vgl. Julia ENCKE, Augenblicke der Gefahr. Der Krieg und die Sinne, 1914-1934, Paderborn, Wilhelm Fink, 2006, S. 152-154. Encke schreibt, dass „,mit dem Eintritt des Stellungskriegs auch ein neues Kapitel der Ohrenheilkunde“" aufgeschlagen worden sei, ibid., S. 153. 
manchmal spitz zusammengefaltet in die Höhe. Sie wird schwächer, stirbt ganz einsam. Eine andere Fliege läuft hin und wieder weg." (T, 310)

Dramatischer und erzähltechnisch komplexer ist die folgende Szene:

\begin{abstract}
Feu ..... er - Alles läuft in Deckung; hinter dem Haus wird ein Stein gesprengt für den Bau der Kommandobaracke. Ein Regen wischt mit den ersten Strichen naß über das Gras. Unter einem Strauch am anderen Bachufer brennt ein Feuer. Daneben steht wie ein Zuseher eine junge Birke. An diese Birke ist mit einem in der Luft hängenden Bein noch das schwarze Schwein gebunden. Das Feuer, die Birke und das Schwein sind allein [Nachtrag:] Und eine lange Blutlache wie eine Fahne.

Dieses Schwein hat schon geschrien, als es ein Einzelner am Stricke führte und ihm zuredete weiter zu kommen. Dann schrie es lauter, als es zwei auf sich zurennen sah. Erbärmlich als es bei den Ohren gepackt und gezerrt wurde. Der Schmerz zog es sprungweise. Am anderen Ende der Brücke griff der eine nach der Hacke und schlug mit der Schneide auf die Stirn. In diesem Augenblick brachen die Vorderbeine gleichzeitig ein. Schrei erst wieder, als das Messer in der Kehle stack [!]. Zucken, Röcheln; wie ein pathetisches Schnarchen. (T, 311)
\end{abstract}

Die Szene setzt mit dem Ruf „Feuer“ ein, der im Krieg wie im Frieden Gefahr bedeutet, sich im gegebenen Fall aber nur auf eine vergleichsweise harmlose Sprengung bezieht. Da die Aufmerksamkeit nun aber geweckt ist, schweift der Blick des Beobachters zum gegenüberliegenden Ufer, wo ein Feuer brennt. Hüben und drüben also Feuer. Auf dieser Seite befindet sich ein Zuseher, aus dessen Perspektive wir die Szenerie mitbetrachten, auf der anderen Seite erkennt man eine junge Birke, die ebenfalls „wie ein Zuseher“ dasteht. Der zwischen den beiden Ufern fließende Bach verstärkt den Eindruck der Spiegelbildlichkeit und ermuntert den Leser, auf beiden Seiten des Wasserlaufs nach weiteren kommunizierenden Elementen zu suchen, die sich sicherlich finden ließen. Über den Bach führt eine Brücke, über die das man kann es nicht anders sagen - arme Schwein gezerrt und gezogen wurde, um dann erst am jenseitigen Ufer hingeschlachtet zu werden. Es ist nicht auszuschließen, dass Musil dem Verdrängungs- und Verschiebungsprozess, dem sein Schreiben über den Krieg unterliegt, mit dieser Szene einen bildlichen Ausdruck gegeben hat.

„Das Feuer, die Birke und das Schwein sind allein“. Man darf vermuten, dass dieser Satz nicht nur um des hübschen Binnenreimes willen formuliert wurde. Die Frage nach dem Verbleib der Schlächter ist also berechtigt. Vielleicht sind auch sie in Deckung gerannt, als sie den Ruf „Feuer“ und gleich darauf die Explosion am anderen Ufer des Baches gehört haben. Vielleicht haben sie - was der Text nicht sagt - zuerst das schwarze Schwein getötet, es dann an einem Bein an der Zuseher-Birke aufgehängt, haben dann unter einem Strauch das Feuer gemacht, um das Schwein darauf zu braten und sind dann erst verschwunden. In jedem anderen Fall, wenn der Schweinekadaver nicht irgendeiner vernünftigen Bestimmung zugeführt hätte werden sollen, hätte es sich um ein sinnloses Schlachten gehandelt, das wohl als Hinweis auf den Krieg zu deuten wäre, so wie auch der dem Text nachträglich hinzugefügte Vergleich der „lange[n] Blutlache“ mit einer 
„Fahne“. Aber man soll Texten keine Gewalt antun, auch dann nicht, wenn sie von Gewalt und - vielleicht - auf verdeckte Weise von den Gräueln des Krieges handeln.

Die markanteste Verschiebung vom Massensterben an der Kriegsfront auf einen wahrnehmungstechnisch leichter $\mathrm{zu}$ bewältigenden Bereich der Erfahrungswelt ist jene, die Musil im Dezember 1917 unter dem Stichwort „Begräbnis in A“ $(T, 337)$ notiert. „A“ steht für Adelsberg, slowenisch Postojna, wo sich das Kommando der k.u.k. Isonzo-Armee befand, dem Musil im Mai 1917 zugeteilt worden war. Markant ist dieser Prozess hier weniger wegen des Ereignisses, das den manifesten Schreibanlass liefert, als vielmehr wegen der besonderen geschichtlichen Bedingungen, unter denen die Verschiebung stattfindet. Karl Corino zitiert aus der historischen Forschung, wenn er schreibt:

\begin{abstract}
Wer annimmt, das [...] Generationserlebnis der Materialschlacht habe man nur in Frankreich erleben können, irrt. Am 23. Mai $1917^{19}$ stand an der Isonzofront alle zehn Meter ein Geschütz oder ein schwerer Minenwerfer. Zwischen sechs Uhr morgens und sechzehn Uhr explodierte eine Million Granaten in den österreichischen Stellungen, rechnerisch 56 Geschosse pro Frontmeter. [...] Es gehörte zur blutigen Mechanik der Isonzokämpfe, daß jedes Remis bald zum Anlass einer neuen Schlacht wurde. Der zehnten folgte die elfte, und der größte Gewinner war erneut der Tod. ${ }^{20}$
\end{abstract}

Als die Zahl der Opfer auf beiden kriegführenden Seiten in die Hunderttausende ging, hat die einzige Tagebuchnotiz, die von Musils Präsenz im Kampfgebiet zeugt, das Begräbnis einer unbekannten Zivilperson zum Thema, deren Tod in keinem erkennbaren Zusammenhang mit den Feindseligkeiten steht. „Es ist fast zu Thränen rührend“, notiert Musil, der vom Fenster seines Zimmers aus den Beginn des Zeremoniells verfolgt. Zum ersten und einzigen Mal in seinem Kriegstagebuch registriert er auch den Geruch des Todes. Der weht aber nicht von den Schlachtfeldern her, sondern kommt, wie er sich beeilt zu bemerken, aus dem Inneren des Hauses, das „erfüllt“" ist „vom Gestank verwester Mäuse“ (T, 337).

Das Sterben einer Fliege, die Tötung eines Schweins, eine um einen einzelnen Sarg versammelte zivile Trauergesellschaft, der Leichengeruch von Mäusen - all diese zu Papier gebrachten Wahrnehmungen beziehen ihre Extravaganz nicht aus sich selbst, sondern aus dem Kontrast zu den welthistorischen Ereignissen, in den sie gesetzt sind. Es sieht so aus, als habe sich Musil in seinen Tagebuchnotizen mit den Begriffen, die ihm zur Verfügung standen, auf die Schilderung des Schilderbaren beschränkt. Etwa im Sinne der These von Helmut Lethen, dass die „vom Dröhnen der vorgeschriebenen Diskurse erschöpfte Wahrnehmung [...] sich an

\footnotetext{
${ }^{19}$ Das Datum markiert den Beginn der 10. von 12 Isonzoschlachten, der zweiten von vier Schlachten, an denen Musil teilnahm.

${ }^{20}$ CORINO, Musil, S. $572 \mathrm{f}$.
} 
bedeutungslosen Dingen [regeneriert]. ${ }^{21}$ Musil selbst hatte an diesem Diskurs teilgehabt und im Chor mit vielen anderen eine glorreiche Wirklichkeit suggeriert, die von den Erfahrungen des Kriegs weggefegt wurde.

\section{Vergrößerung}

Befragt man Musils Texte nach einer übereinstimmenden Formel, die er diesen Erfahrungen abringen konnte, so löst sich aus den Skizzen und Aufzeichnungen des Tagebuchs, aus den in den Kriegsjahren verfassten Prosafragmenten sowie aus den rückblickenden Betrachtungen der späteren Jahre die Vorstellung der Vergrößerung. Der Krieg habe viele Phänomene, die schon vor 1914 da waren, verstärkt und damit augenscheinlicher gemacht. Vom Helden des kleinen Prosatextes mit dem zögernden Titel Der Gesang des Todes/ Der singende Tod, einem Maler und Ingenieur, dem der Autor ganz offenkundig autobiographische Züge verliehen hat, von diesem Helden heißt es etwa: „Der Mann, von dem das erzählt wird, hatte bald den Eindruck, dass er in den Krieg gegangen war, wie man vor ein ungeheures Vergrößerungsglas tritt.“ (PS, 758)

Dass die Zustände vor und nach dem Ausbruch des Weltkriegs nicht grundsätzlich verschieden waren, sondern manche strukturellen Gemeinsamkeiten aufwiesen, hat auf seine Art auch Karl Kraus zum Ausdruck gebracht, wenn er in den Letzten Tagen der Menschheit den Nörgler sagen lässt, dass der barbarischen deutschen Kriegführung eine barbarische deutsche Friedensführung vorangegangen sei. ${ }^{22}$ An anderer Stelle heißt es, weniger polemisch und mehr im Sinne Musils, der Krieg sei „ein Anschauungsunterricht durch stärkere Kontrastierung.“233

Was da durch den Effekt der Vergrößerung oder der Kontrastierung stärker zutage tritt, ist dem Bereich der Materialschlachten zuzurechnen, vor allem dann, wenn man die Grenze des Begriffs Material - in Einklang mit den militärstrategischen Gepflogenheiten der Zeit - so weit lockert, dass nicht nur der Einsatz moderner Waffen, sondern auch das durch diese Waffen verstümmelte und getötete Menschenmaterial in ihm Platz findet.

Das häufig beschriebene Einheitsgefühl der Masse Mensch, das anlässlich der Mobilisierung freigesetzt wurde, konnte sich Alexander Honold zufolge, auch deshalb zu rauschhafter Intensität steigern, weil es als „Umkehrung

\footnotetext{
${ }^{21}$ Helmuth LETHEN, Verhaltenslehren der Kälte. Lebensversuche zwischen den Kriegen, Frankfurt am Main, Suhrkamp, 1994, S. 187.

22 „Aber wenn die andern sagen, die deutsche Kriegführung sei barbarisch, so fühlen sie doch mit Recht, dass die deutsche Friedensführung barbarisch ist. Und das muß sie gewesen sein, da sie sonst nicht seit Generationen auf dem Gedanken aufgebaut gewesen wäre, die deutsche Kriegführung vorzubereiten“, Karl KRAUS, Die letzten Tage der Menschheit. Tragödie in fünf Akten mit Vorspiel und Epilog, Teil I, München, dtv, 1975, I, 29, S. 150.

${ }^{23}$ Ibid., I, 22, S. 118.
} 
realer Trennungen“ begrüßt wurde, „die durch Arbeitsteilung, durch ökonomische und politische Konfliktlinien [...] markiert waren.“ ${ }^{\text {“24 }}$ Ähnlich argumentiert Klaus Vondung, wenn er meint, der Krieg habe der „zunehmenden[n] Desintegration der Gesellschaft in die zwei Nationen von Adel und Bürgertum einerseits und der Arbeiterschaft andererseits" vorübergehend entgegengewirkt. ${ }^{25}$ Dass diesen Gefühlen und Tendenzen unter den Bedingungen des ersten Weltkriegs aber oft keine Dauer gegeben war, erklärt Sigmund Freud in der Schrift Massenpsychologie und IchAnalyse (1921) mit der „libidinöse[n] Struktur“ des Heeres. Wie auch in der Kirche wirke die libidinöse Energie in zwei Richtungen - in horizontaler und in vertikaler -, wobei über die horizontale Wirkung der Zusammenhalt unter den Massenindividuen gesichert werde. Entscheidend sei in diesem System aber das, was von oben komme:

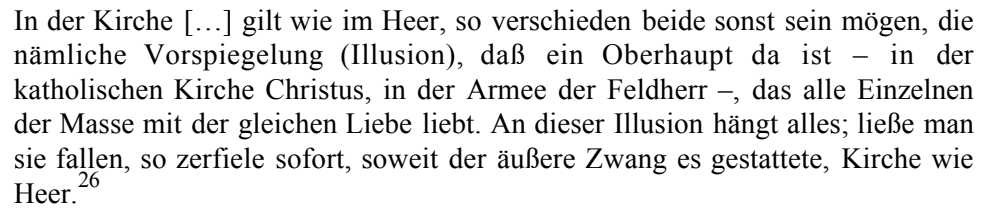

In der Kirche [...] gilt wie im Heer, so verschieden beide sonst sein mögen, die nämliche Vorspiegelung (Illusion), daß ein Oberhaupt da ist - in der katholischen Kirche Christus, in der Armee der Feldherr -, das alle Einzelnen der Masse mit der gleichen Liebe liebt. An dieser Illusion hängt alles; ließe man sie fallen, so zerfiele sofort, soweit der äußere Zwang es gestattete, Kirche wie Heer. ${ }^{26}$

Freud sieht in der libidinösen Bindung an den Feldherrn nicht den einzig wirksamen Faktor bei der Massenbindung in der Armee, hält ihn aber für bedeutender als etwa die „Ideen des Vaterlandes“ oder „,des nationalen Ruhmes.“27 Der preußische Militarismus habe nun mit seiner „lieblosen Behandlung des gemeinen Mannes“ dessen Libidoansprüche nachhaltig enttäuscht, was $\mathrm{zu}$ zahlreichen, die deutsche Armee zersetzenden Kriegsneurosen geführt und das Gemeinschaftsgefühl geschwächt habe. ${ }^{28}$

Wenn man dem Freudschen Modell des vertikalen und horizontalen Libidostromes auch nur ein wenig Gültigkeit beimisst, wird man die negativen Konsequenzen, die sich aus einer gestörten Verbindung zwischen Soldat und Kommando für das Einheitsgefühl der militärischen Masse ergeben, kaum hoch genug veranschlagen können. Man geht wohl nicht fehl in der Annahme, dass die Situation der österreichisch-ungarischen Soldaten nicht wesentlich anders war als die ihrer deutschen Waffenbrüder. Jedenfalls

${ }^{24}$ HonOLD, Die Stadt und der Krieg, S. 195.

${ }^{25}$ Vgl. Klaus Vondung, Die Apokalypse in Deutschland, München, dtv, 1988, S. 198 f.

26 Sigmund FrEUD, „Massenpsychologie und Ich-Analyse“, in Sigmund FreUD, Studienausgabe, Band IX, Fragen der Gesellschaft, Ursprünge der Religion, Frankfurt am Main, Fischer, 1974, S. 88 f.

Zum libidinösen Verhältnis zwischen den gemeinen Soldaten einerseits, zwischen den gemeinen Soldaten und den Kommandierenden andererseits vgl. auch Klaus THEWELEIT, Männerphantasien, 1. Frauen, Fluten, Körper, Geschichte, Reinbek bei Hamburg, Rowohlt, 1980, S. 66-71.

${ }^{27}$ Vgl. ibid., S. 89

${ }^{28}$ Vgl. ibid., S. 89 f. 
schreibt Musil über den Verlust dieses Einheitsgefühls, über dessen Umkehrung ins Gegenteil, in einem Essayentwurf aus dem Jahre 1918:

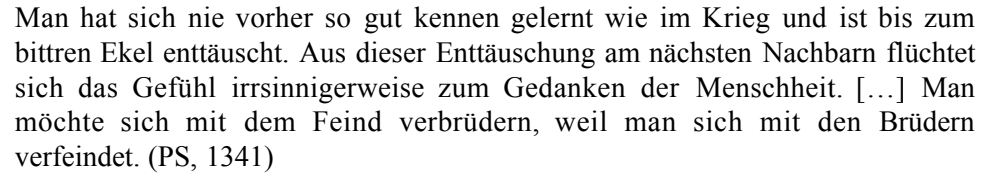
bittren Ekel enttäuscht. Aus dieser Enttäuschung am nächsten Nachbarn flüchtet sich das Gefühl irrsinnigerweise zum Gedanken der Menschheit. [...] Man möchte sich mit dem Feind verbrüdern, weil man sich mit den Brüdern verfeindet. (PS, 1341)

Dass die vertikale Verbindung häufig nicht nur gestört, sondern vollkommen anonym geworden war, erkannte etwa Krupp-Direktor Freiherr von Bodenhausen, der 1915 vom „Fabrikbetrieb des modernen Krieges“ sprach, ,in dem der Commandierende seine Truppen nicht kenne, sehe, oder mit ihnen Fühlung habe", und der meinte, dass aus den Planspielen der Strategen unter den Voraussetzungen des „modernen - nämlich industriellen - Krieges [...] vollständige Bureauarbeit" geworden sei. ${ }^{29}$ Das Zitat ist nicht nur deshalb interessant, weil von berufener Seite die Verbindung zwischen der Führung und dem soldatischen Fußvolk als gekappt begriffen wird, sondern auch weil das verwendete Vokabular („Fabrikbetrieb“, „Bureauarbeit") deutlich macht, wie weit die Entmystifizierung des Krieges zumindest bei Krupp - schon gediehen war. Aus dem gleichen Wortfeld schöpft auch Peter Sloterdijk, wenn er schreibt, seinem Wesen nach sei der „namenlose[] Held“ des Ersten Weltkriegs ,doch mehr Techniker als Kämpfer, mehr Staatsangestellter als Heros“" gewesen. ${ }^{30}$

Solche Befunde weisen darauf hin, dass ein Volk, das herrlichen Zeiten entgegengeführt werden sollte, in diesen angekommen oder auf dem Weg dahin, soziale Strukturen und Verhältnisse vorfand, die denen ähnelten, die es im Sommer 1914 so freudig verlassen hatte. Der Effekt der „Vergrößerung“, der Akzentuierung, betrifft hier die Rahmenbedingungen, unter denen diese Verhältnisse nun gelebt werden mussten und die kriegsbedingt ungleich schwieriger geworden waren. Die Sachlichkeit in den menschlichen Beziehungen, die sich innerhalb der technisierten Massengesellschaft entwickelt hatte, bekam durch die täglich erwiesene Möglichkeit des Todes einen verschärft zynischen Charakter. So heißt es bei Musil:

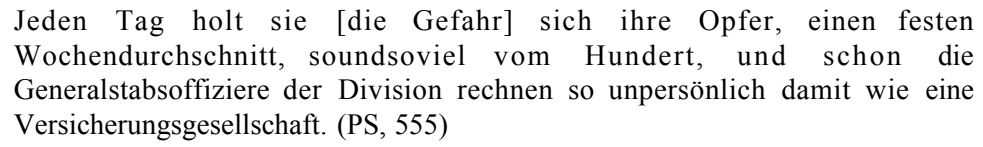

\footnotetext{
${ }^{29}$ Zitiert nach: Peter SPRENGEL, ,'Im Kriege erscheint Kultur als ein künstlicher Zustand'. Gerhart Hauptmann und der Erste Weltkrieg“, in Uwe SCHNEIDER und Andreas SCHUMANN (Hrsg.), Krieg der Geister. Erster Weltkrieg und literarische Moderne, Würzburg, Königshausen \& Neumann, 2000, S. 59.

${ }^{30}$ Peter SLOTERDIJK, Kritik der zynischen Vernunft, Zweiter Band, Frankfurt am Main, Suhrkamp, 1983, S. 414.
} 


\section{Der ,lebendige Tod“"}

Statt Teil eines sinnvollen Ganzen war man - das scheint zumindest Musils Grundstimmung gewesen $\mathrm{zu}$ sein - mit einem ungeliebten Massenkörper verwachsen, dessen Bewegungen man willenlos mit auszuführen hatte. Eine der ganz wenigen Skizzen zu einer Schlachtszene beschreibt dies mit knappen Worten:

[...] aus dem Eisenbahnwagen stieg man ins Artilleriefeuer, hastete verbissen vorwärts, um endlich ans Ende dieser verzweifelten Schießerei zu gelangen, wurde plötzlich in Splitter zerrissen, Flucht, flog als Wolke zurück, wurde gesammelt, wieder vorgeführt, unterlag oder siegte, ohne es zu wissen. (PS, 757)

In den „Wellenbrecherschlachten“ (ibid.) des Ersten Weltkrieges wurde der Einzelne auf drastische Weise auf eine der Grunderfahrungen der Moderne zurückgeworfen, nämlich einen großen Teil der beanspruchten Entscheidungsgewalt über sein Leben eingebüßt zu haben. Der im Laufe des 19. Jahrhunderts aufdringlicher gewordene Gedanke, „daß es - irgendwie nicht mehr so sehr auf ihn ankomme“, ist dem Menschen dann, wie Musil 1934 in einer Rede erklärt, ,vom Krieg dazu noch sehr eindringlich doziert worden." (PS, 1246)

Für Clausewitz, der seine Erfahrungen vor allem in den Schlachten gegen Napoleon gesammelt hatte, war der „Krieg [...] nichts als ein erweiterter Zweikampf $^{* 31}$ und mit Abstrichen lässt sich diese Formel auch noch auf den Preußisch-Österreichischen Krieg von 1866 und den Deutsch-Französischen Krieg von 1870/71 anwenden. So hatten die habsburgischen Truppen in der Schlacht von Königgrätz noch versucht, „die Entscheidung mit gefälltem Bajonett im Nahkampf [zu] erzwingen“, zu dem es der technologisch überlegene Gegner aber kaum mehr kommen ließ:

Die Preußen belegten sie mit konzentriertem Feuer aus ihren für die damalige Zeit hochmodernen Dreyse-Gewehren, mit Zündnadeln versehenen Hinterladern, die fünf Schüsse in der Minute abgeben konnten. Die veralteten Vorderlader der österreichischen Armee [...] schafften höchstens einen Schuss in der Minute. [...] Die tapfere Truppe [...] des Vielvölkerstaates [...] marschierte mannhaft in das mörderische Feuer.

31 Zitiert nach Christian STADLER, Krieg, Wien, Facultas, 2009, S. 80. 522.

Stephan VAJDA, Felix Austria. Eine Geschichte Österreichs, Wien, Ueberreuter, 1980, S.

Dieses Zitat macht auch deutlich, dass die seit Königgrätz sich hartnäckig behauptende Vorstellung, die österreichischen Soldaten seien „fesch, aber feig“, nur zu Hälfte richtig ist. Die „,in ihren auffälligen, farbenprächtigen Monturen schon von weitem sichtbar[en]“ Truppen Franz Josephs waren weniger feig' als vielmehr technisch und strategisch rückständig.

Vgl. Ödön VON HoRVATH, Geschichten aus dem Wiener Wald, Gesammelte Werke, Bd. 4, Frankfurt am Main, Suhrkamp, 1986, S. 61 und 162.

Vgl. VAJDA, Felix Austria, S. 522. 
Paul Virilio sieht im Waffengang von 1914-1918 den „erste[n] mediatisierte[n] Krieg der Geschichte [...], in dem Schnellfeuerwaffen an die Stelle der Vielzahl individueller Waffen traten“, und der Kampf „Mann gegen Mann [...] seine grundsätzliche Bedeutung [verlor]. ${ }^{633}$ Daniel Marc Segesser schreibt, dass die „meisten Soldaten [...] während des Stellungskrieges nicht wegen einer direkten Feindberührung ums Leben [kamen].“ Der weitaus größte Teil „der Verluste war vielmehr auf Artilleriebeschuss zurückzuführen. ${ }^{\text {(34. }}$.

Musils Tagebuchaufzeichnungen zeugen davon, dass das Massensterben zu den sinnlich und wohl auch mental kaum mehr nachvollziehbaren Eindrücken des Ersten Weltkriegs gehört. Dem anonymen soldatischen Massenkörper - so lautet die ständig wiederholte Lektion der Kampfhandlungen - konnte man letztlich nur als Toter entkommen, wenn man nach dem Zufallsprinzip aus der Schicksalsgemeinschaft herausgeschossen wurde und „wahnsinniges Feuer des Gegners [...] Löcher mit blutigen Rändern in die Masse ${ }^{\text {‘35 }}$ riss. Auf diesen Tatbestand reagiert der Soldat Musil mit dem Bedürfnis nach Isolation und der Sehnsucht nach einem individuellen Sterben, wie es auch in der oben zitierten Skizze „Begräbnis in $\mathrm{A}^{\text {“ zum }}$ Ausdruck kommt. Nun schreibt er davon, manchmal „ohne äußeren Grund“ und ,allein [...] z.B. morgens bei klarer Sicht, wenn alles in den Deckungen steckt, spazieren [zu] gehen." Er ist sich der Gefahr, in die er sich dabei begibt, wohl bewusst, und „bei jedem Schritt [ist] eine gewisse Überwindung nötig." Hier, zwischen den eigenen und den feindlichen Stellungen, scheint der Tagebuchschreiber eine Situation provozieren zu wollen, in der der einzelne Soldat noch einmal als solcher identifizierbar und der „Tod etwas ganz Persönliches [ist]“. Bei dem „Glücksgefühl“, das ihn dabei „überströmt", $(T, 325)$ handelt es sich offenbar um eine Reminiszenz an ein Erlebnis, das seine volle Wirkung nicht im Augenblick des Geschehens, sondern erst im Nachhinein entwickelt hat und über das er später schreibt: ,jedesmal, wenn ich mich daran erinnere, möchte ich etwas von dieser Art noch einmal deutlicher erleben!“ (PS, 557)

Am 22. September 1915 notierte er unter dem Titel „Das Schrapnellstück oder der Fliegerpfeil auf Tenna“, dass ein Fliegerpfeil unmittelbar neben ihm in den Boden einschlug und er dem Tod nur dadurch entgangen war, dass er seinen „Oberkörper zur Seite [riß] und [...] bei feststehenden Füßen eine ziemlich tiefe Verbeugung [machte]." Der Begebenheit wird sofort eine ins

\footnotetext{
${ }^{33}$ Zitiert nach Arno RuSSEGGER, ,'Daß Krieg wurde, werden musste, ist die Summe all der widerstrebenden Strömungen und Einflüsse und Bewegungen, die ich zeige'. Erster Weltkrieg und Moderne - am Beispiel von Robert Musil, in ScHNEIDER / SchumAnN, Krieg, S. 241. Das Originalzitat in Paul VIRILIO, Guerre et cinéma I. Logistique de la perception, Paris, Cahiers du cinéma, nouvelle édition augmentée, 1991, S. 123.

${ }^{34}$ Vgl. Daniel Marc SEgesSER, Der Erste Weltkrieg in globaler Perspektive, Wiesbaden, marixverlag, 2012, S. $128 \mathrm{f}$.

${ }_{35}$ Musil in der Tiroler Soldaten-Zeitung vom 26. Juli 1916. Zitiert nach CORINO, Musil, S. $561 \mathrm{f}$
} 
Religiöse weisende Bedeutung beigemessen: „Befriedigung, es erlebt zu haben. Beinahe Stolz; aufgenommen in eine Gemeinschaft, Taufe.“ (T, 312)

In insgesamt fünf überlieferten Anläufen hat Musil diesem Erlebnis eine sprachliche Form zu geben versucht und es schließlich als mittlere von drei Episoden in die 1928 erschienene Novelle Die Amsel integriert. „Fliegerpfeil[e], heißt es dort erläuternd,

waren spitze Eisenstäbe, nicht dicker als ein Zimmermannsblei, welche damals die Flugzeuge aus der Höhe abwarfen; und trafen sie den Schädel, so kamen sie wohl erst bei den Fußsohlen wieder heraus, aber sie trafen eben nicht oft, und man hat sie bald wieder aufgegeben. (PS, 555)

Die teilweise befiederten, etwa 10 bis $15 \mathrm{~cm}$ langen Eisen- oder Stahlstifte, markierten „,den Übergang von archaischen zu modernen Waffentechniken. ${ }^{\text {‘36 }}$ Modern war neben dem Abwurf aus einem Flugzeug vor allem die strategische Umfunktionierung der „intentionale[n]“ oder gerichteten „Waffe zu einer statistischen ${ }^{\text {(37 }}$, die immer dann vorlag, wenn Fliegerpfeile ,in großer Menge herabgeschleudert“ wurden. ${ }^{38}$ Die auch im gewöhnlichen Leben stets vorhandene Möglichkeit eines plötzlichen Todes wurde dann ,in ungeheurer Vergrößerung“ erfahren. Der

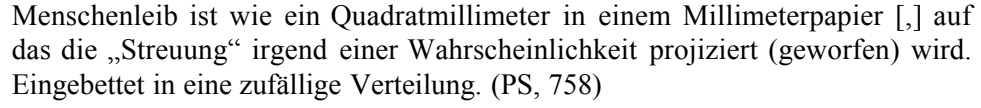

An die Stelle des ,schauerliche[n] Gerippe[s], das man täglich an uns vorbeitrug“, an die Stelle des „wahnsinnige[n] [,] zweckbefangene[n], geschäftige[n] Tod[es]", der die Soldaten unter diesen und ähnlichen Bedingungen trifft, rückt in Musils Wunschvorstellung „der lebendige, der geistliche Tod“, der „nur an stilleren Fronten zuhause ist.“ (PS, 752) Als solche gestaltet der Autor ansatzweise jenen Ort, an dem er das FliegerpfeilErlebnis in seiner literarischen Verarbeitung situiert und in dessen Beschreibung sich biblisch-archaisierende Wendungen mengen. Die Truppe hielt damals im „toten Winkel einer Kampflinie in Südtirol“ (PS, 554) eine vorgeschobene Position. „Von Gott wie ein Posaunenstoß geschaffen“ (PS, 752), lag das Suganer Tal in der Herbstsonne und die Stellung des Bataillons war darin ,so offen [...], dass man uns von oben mit Steinwürfen erschlagen konnte." (PS, 554)

Der „lebendige Tod" tritt aber nicht auf diese althergebrachte Weise an die Soldaten heran, sondern nähert sich „einmal mitten in der Zeit“ durch einen feindlichen Flieger. Die

\footnotetext{
${ }^{36}$ RUSSEGGER, Krieg, S. 240.

${ }^{37}$ ENCKE, Augenblicke, S. 175.

${ }^{38}$ Vgl. CoenEN, „Der Pfeil als Fliegerwaffe“, in Berliner Klinische Wochenschrift, Jg. 51, Nr. 43, 26.10.1914, S. 1745. Zitiert nach ENCKE, ibid.
} 
Sonne schien durch die dreifarbigen Tragflächen des Flugzeugs, gerade als es hoch über unseren Köpfen fuhr wie durch ein Kirchenfenster oder buntes Seidenpapier, und es hätte zu diesem Augenblick nur noch einer Musik von Mozart bedurft. (PS, 555)

Der Hinweis auf Mozart ist ein deutliches Indiz für ein bevorstehendes, die Grenzen der alltäglichen Erfahrung sprengendes Ereignis. ${ }^{39}$ In vier der fünf vorliegenden Textvarianten hört der Ich-Erzähler einen Laut. Im Tagebuch ist es ein „windhaft pfeifendes oder rauschendes Geräusch“ (T, 312), in den literarischen Verarbeitungsstufen „ein leises Singen“ (PS, 752, 755), „ein leises Klingen“ (PS, 555), dann „ein hoher, dünner, singender[,] einfacher Laut“ (PS, 755) oder auch ein „Laut [...] wie der einer Stimmgabel“, an dem „,vom ersten Augenblick an etwas Unirdisches [war] (PS, 753), der das „Musikhafte“ aber nicht verlor (vgl. PS, 753). Und immer ist da auch die Gewissheit: „es ist ein Fliegerpfeil!“ (PS, 555) Und fast stärker als diese die sichere Erkenntnis, er „war auf mich gerichtet“ (PS, 556,756) sowie „das sonderbare, nicht im Wahrscheinlichen begründete Empfinden: er trifft" (PS, 556)! An diese - kriegsgeschichtlich gesehen - anachronistischen Gewissheiten knüpft sich die den realen Lebens-bedingungen trotzende Vorstellung des ,persönliche[n] Auserwähltsein[s] trotz der Statistik.“ (PS, 754)

Der Ich-Erzähler glaubt, „eine persönliche Weihe empfangen zu haben“ (PS, 753), fühlt „Gottes Nähe“ (PS, 556) und es fällt ihm ein: „,so ist es, wenn Gott etwas verkünden will“ (PS, 756). Die religiöse Überhöhung der Szene ist angesichts der geschilderten Eindrücke nicht verwunderlich, denn es drückt sich in ihr, wie Dietmar Goltschnigg in anderem Zusammenhang erläutert, „die bekannte mystische Erfahrung aus, dass Gott gerade dann dem Menschen am nächsten zu stehen scheint, wenn dieser sich selbst, sein eigenes Ich erlebt ${ }^{\star 40}$.

Für den Menschen, dem in modernen Friedenszeiten schon längst das Verschwinden in der anonymen Masse droht, bedeutet der Erste Weltkrieg eine extreme Zuspitzung dieser krisenhaften Situation. Auf dem Schlachtfeld, im Augenblick der intuitiv erkannten Gefahr, streift den Soldaten Robert Musil die Ahnung von der Möglichkeit eines persönlichen Schicksals. Im literarischen Text lässt der Autor Robert Musil die ersehnte Individuation schließlich noch einmal gelingen.

\footnotetext{
${ }^{39}$ Aus dem Wortfeld Musik stammende Begriffe werden von Musil häufig als fester, verselbständigter Tropus verwendet. Gradischnig erkennt in der Vermittlung durch Musik ein Strukturelement der für Musils Helden charakteristischen Erweckungserlebnisse. Vgl. GRADISCHNIG, Das Bild ..., S. 74, 85, 95. Vgl. zu dieser Thematik auch Ernst KAISER, Eithne WILKINS, Robert Musil. Eine Einführung in das Werk, Stuttgart, Kohlhammer, 1962, S. 101.

${ }^{40}$ Dietmar GolTSCHNIGG, Mystische Tradition im Roman Robert Musils. Martin Bubers ,Ekstatische Konfessionen' im ,Mann ohne Eigenschaften' “, Heidelberg, Lothar Stiehm, 1974, S. 60 .
} 